\title{
Quantum Dimer Model on the Kagome Lattice: Solvable Dimer Liquid and Ising Gauge Theory
}

\author{
G. Misguich, f D. Serban, and V. Pasquier \\ Service de Physique Théorique, CEA/Saclay 91191 Gif-sur-Yvette cédex, France
}

\begin{abstract}
We introduce quantum dimer models on lattices made of corner-sharing triangles. These lattices includes the kagome lattice and can be defined in arbitrary geometry. They realize fully disordered and gapped dimer-liquid phase with topological degeneracy and deconfined fractional excitations, as well as solid phases. Using geometrical properties of the lattice, several results are obtained exactly, including the full spectrum of a dimer-liquid. These models offer a very natural - and maybe the simplest possible - framework to illustrate general concepts such as fractionalization, topological order and relation to $\mathbb{Z}_{2}$ gauge theories.
\end{abstract}

PACS numbers: 75.10.Jm 75.50.Ee 74.20.Mn

Quantum dimer models (QDM) were introduced by Bokhsar and Kivelson [1] in the context of resonating valence-bond (RVB) theories for the high-temperature su"iperconductors [2]. Such models are expected to describe the dynamics of singlet bonds (dimer) in quantum disordered sspin- $\frac{1}{2}$ antiferromagnets. They can describe two generic ghases: spin-liquids where the system breaks no symmetry at all and dimer (or valence-bond) crystals where long-range Gimer-dimer correlations develops. Recently a genuine liqFid phase with a finite correlation length was found in a QDM by Moessner and Sondhi on the triangular lattice 3 . Stuch liquid states have attracted a lot of interest because they display both fractional excitations and topological order [4. While fractionalization could play an important role cin some theories of high-temperature superconductors [2, 5, the topological properties of these liquid states have been proposed as possible devices to implement quantum bits for quantum computations [6, 07.

In this Letter we introduce QDM which realize such a dimer-liquid phase. Due to the geometric properties of lattices made of corner-sharing triangles (the simplest twoGimensional example being the kagome lattice [22]), these models are solved exactly. As for the solvable point of the QDM on the square [1] and triangular [3] lattices (see also Refs. [8] and [9]), the ground-state is the equal-amplitude superposition of all dimer coverings in a given topological esector 23. Such state has been first considered by SutherHand 10] (SRK state in the following) and is the prototype of Tesonating valence-bond (RVB) state. However, our model has several important differences with previous analogs : 1) Jot only the ground state but all excited states wave functiōns are known. Elementary excitations are (pairs of) non interacting and gapped vortices 11] (called visons in the recent literature [5]). 2) The model can be solved on any geometry: torus, discs or spheres. This allows to investigate the interplay between topology, ground-state degeneracy and elementary excitations in an very simple way. 3) Dimer-dimer correlations are strictly zero above one lattice spacing. This makes this SRK wave function the most possible disordered dimer liquid state. 4) This state is inside the liquid phase, it is not lying at the phase boundary with a crystalline phase.

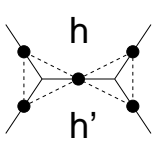

FIG. 1: Medial lattice construction. From a trivalent lattice $H$ (full lines) we construct a lattice $K$ whose sites (black dots) are centers of the bonds. The sites of $K$ are linked together (dashed lines) to form triangular plaquettes. The pseudospin variables live on the dual of $H$ ( $\mathrm{h}$ and $\mathrm{h}$ ').

5) It is known 12 that QDM can be obtained as special limits of $\mathbb{Z}_{2}$ gauge theories, the gauge variable being the dimer number on a bond. Here we show a complete equivalence with a $\mathbb{Z}_{2}$ gauge theory. This allows to investigate the confinement transition which goes with a dimer crystallization in a simple QDM which exhibits a liquid to solid transition accompanied by a vison condensation.

For all these reasons the model we introduce is more than a toy model but the simplest possible RVB liquid. It is a "free dimer liquid" point in the short-ranged RVB phase. It shows the generic properties which characterize that state of matter but almost any quantity can be computed exaclty. It is a natural starting point for perturbative expansions toward more realistic models.

Medial lattice construction.- The dimer models described in this Letter can be defined on lattices made of cornersharing triangle, constructed in the following way. Let $H$ be a trivalent lattice (each site has three neighbors). The lattice $K$, where the dimers live, is the medial lattice of $H$, i.e. the sites of $K$ are the midpoints of the bonds of $H$ (Fig. 1). If $H$ is the honeycomb lattice, $K$ is the kagome lattice (Fig. 2a). In the following, unless mentioned otherwise, we use kagome for simplicity, where plaquettes of $H$ are hexagons. We stress however that all results can be generalized straightforwardly to other lattices (squagome [16] lattice for instance, as well as one-dimensional examples).

Pseudospin representation. - Let us begin with the definition of a simple dimer model. For each hexagon $h$, we define an operator $\sigma^{x}(h)$ as the sum of all possible kinetic energy 
terms involving $h$ only:

$$
\sigma^{x}(h)=\sum_{\alpha=1}^{32}\left|d_{\alpha}(h)\right\rangle\left\langle\bar{d}_{\alpha}(h)|+| \bar{d}_{\alpha}(h)\right\rangle\left\langle d_{\alpha}(h)\right|
$$

The sum runs over the 32 loops on kagome which enclose a single hexagon and around which dimers can be moved (see Ref [15] for an explicit list). The shortest loop is the hexagon itself, it involves 3 dimers. 4, 5 and 6 -dimers moves are also possible by including 2, 4 and 6 additional triangles (the loop length must be even). The largest loop is the star. For each loop $\alpha$ we associate the two ways dimers can be placed along that loop: $\left|d_{\alpha}(h)\right\rangle$ and $\left|\bar{d}_{\alpha}(h)\right\rangle$.

For a given dimer covering $|D\rangle$, all the kinetic operators in the sum but one annihilate $|D\rangle$. As a consequence, $\sigma^{x}(h)^{2}=1$. One can further check that these operators flip the pseudospin variables $\sigma^{z}(h)$ introduced by Elser and Zeng [14, 15] (EZ) to label dimer coverings 24]. It is important to note that $\sigma^{z}$ operators depend on the choice of a reference $\left|D_{0}\right\rangle$ and are not local, unlike $\sigma^{x}$. The $\sigma^{x}(h)$ commute with each other. This is not obvious from Eq. 1 and it is most easily demonstrated in terms of the arrow representation that we introduce below.

Arrow representation.- A correspondence between dimer coverings on the kagome lattice and sets or arrows as illustrated in Fig. 22a was introduced by Elser and Zeng [14. Each arrow has two possible directions: it points toward the interior of one of the two neighboring triangles. If site $i$ belongs to a dimer $(i, j)$ its arrow must point toward the triangle the site $j$ belongs to. Consider a triangle without any dimer, this arrow rule implies that it will have three outgoing arrows. Other triangles will have two incoming arrows and one outgoing arrow. In other words, the number of outgoing arrows is constrained to be odd.

The number of dimer coverings is $2^{N / 3+1}$ where $N$ is the number of sites [13, 14. $K$ has $N$ sites and $N$ arrows, $2 N / 3$ triangles and one constraint per triangle. However only $2 N / 3-1$ constraints are independent because their product for all triangles is equal to one. We are left with $N / 3+1$ Ising degrees of freedom. The existence of this arrow representation is a central reason for which QDM considerably simplify on these lattices. For example $\sigma^{x}(h)$ translates very simply in the arrow representation: it flips the six arrows sitting around hexagon $h$, which clearly conserves the constraint for all triangles and commutes from hexagon to hexagon.

Rokhsar-Kivelson point.- Consider the following Hamiltonian:

$$
\mathcal{H}_{0}=-\Gamma \sum_{h} \sigma^{x}(h)
$$

where the sum runs over hexagons (sites of the dual of $H$ in general). Although very simple in the pseudospin variables, this Hamiltonian is not obviously solvable when written with dimer operators. In the pseudospin variables the ground-state is a fully polarized ferromagnet in the $x$ direction, which is the sum of all pseudospin configurations in the
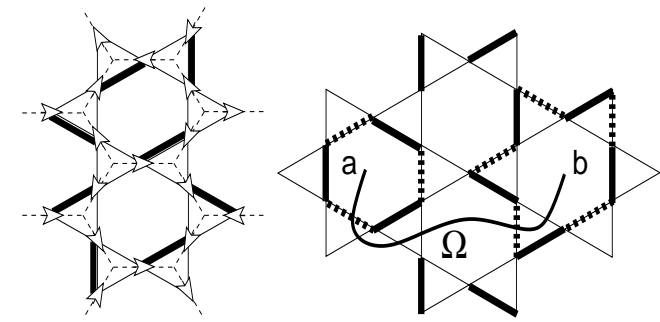

FIG. 2: a) A dimer covering on the kagome lattice (fat bonds). The corresponding arrow representation is displayed. The Kagome lattice is the medial lattice of the hexagonal lattice (dashed bonds). b) A pair of visons is created by applying to the SRK wave-function a factor $(-1)$ for each dimer crossing the cut $\Omega$. It is an exact eigenstate of Eq. 2 .

$\mathrm{EZ} \sigma^{z}$ basis. Back to dimers, this is nothing but the sum of all dimer configurations in a given topological sector, that is a SRK wave-function |1]. The ground-state appears to be unique in each topological sector, which gives a global 4-fold degeneracy on the torus.

Correlations.- Correlations in the SRK state of the kagome lattice are particularly simple: irreducible dimerdimer correlations are strictly zero when their corresponding triangles do not touch. The arrows on two bonds are independent provided they are not involved in a common constraint, that is a common triangle. As a result, dimer on the kagome lattice are the most possible disordered: they are independent above a finite distance.

Gap.- The whole spectrum is known. The $\sigma^{x}$ operators commute from hexagon to hexagon but physical dimer states must satisfy $\prod_{h} \sigma^{x}(h)=1$. This constraint comes from the arrow representation since $\prod_{h} \sigma^{x}(h)$ flips all the arrows twice and therefore keeps all dimerizations unchanged. The first excited state appears not to be a single but a pair of flipped hexagons with energy cost $\Delta=4 \Gamma$.

Visons.- Despite of the simplicity of the model in the pseudospin variables, its excited states are not local when expressed with dimer degrees of freedom. A $\sigma^{x}(h)=-1$ hexagon is a vortex excitation (also called vison [5]). Consider a string which goes from an hexagon $a$ to and hexagon $b$ (see Fig. 2b) and let $\Omega(a, b)$ be the operator which measures the parity \pm 1 of the number of dimers crossing that string. $\Omega(a, b)$ commutes with all $\sigma^{x}(h)$, except for the ends of the string: $\sigma^{x}(a) \Omega(a, b)=-\Omega(a, b) \sigma^{x}(a)$. A dimer move changes the sign of $\Omega(a, b)$ if and only if the associated loop crosses the string an odd number of times, which can only be done by surrounding one end of the string. This shows that $\Omega(a, b)$ flips the $x$ component of the pseudospin in $a$ and $b$ [25] and $\Omega(a, b)|\psi\rangle$ is precisely the excited state of energy $4 \Gamma$ discussed above. Visons appear to be perfectly localized in this model.

Spinons.- One can consider the system with two static unpaired sites (spinons or holes). As for others QDM at a SRK point [1, 3], the sum of all dimerizations in a given sec- 
tor remains an exact eigenstate. The energy turns out to be independent of the relative distance between spinons, which is a strong indication that spinons would be also deconfined if they had kinetic energy. Eventually, we note that taking a spinon around a vison will change the wave function by a -1 factor, as expected 11.

Visons and topology.- On a closed surface of genus $g$, the spectrum has a degeneracy given by the number of topological sectors $2^{2 \mathrm{~g}}$ and excitations are pairs of visons, as already mentioned. When the sample has edges, the constraint $\prod_{h} \sigma^{x}(h)=1$ is not valid anymore. To handle this case we introduce $\sigma^{x}(\tilde{h})$ which flips the arrows along the edge and which restores $\sigma^{x}(\tilde{h}) \prod_{h} \sigma^{x}(h)=1$. The excitations are still pairs of visons but one vison can be located in the hole 5 . In this case the gap reduces to $2 \Gamma$. In an cylinder geometry, as in Ref. [7 for instance, we have two sectors and a doubly degenerate spectrum. It is interesting to note that this dimer liquid has no low-energy edge states.

Liquid - solid transition. - We consider a new QDM which is a generalization of Eq. 2:

$$
\mathcal{H}_{1}=\mathcal{H}_{0}-J \sum_{\left\langle h, h^{\prime}\right\rangle} \sigma^{z}(h) \sigma^{z}\left(h^{\prime}\right)
$$

where the second sum runs over pairs of neighboring hexagons. The $\sigma^{z}$ operators are those defined by EZ, they depend on the choice of a reference dimerization $\left|D_{0}\right\rangle$. A term $\sigma^{z}(h) \sigma^{z}\left(h^{\prime}\right)$ is local, it is $=1$ if the arrow which is in between $h$ and $h^{\prime}$ is in the same position as in the reference state and -1 otherwise. Since $\sigma^{z}(h) \sigma^{z}\left(h^{\prime}\right)$ is equivalent to $\Omega\left(h, h^{\prime}\right)$, we see that such a term allows to create, annihilate and move visons in the system. From another point of view, the $J$ term in Eq. 3 counts the number of arrows to be flipped to recover the reference state. If $J$ goes to $+\infty$, this Hamiltonian obviously selects the reference state as the ground-state and dimers are completely frozen. In the pseudospin language $\mathcal{H}_{1}$ is an Ising ferromagnet in transverse field, which displays a second order phase transition at a critical value of $\Gamma$ separating two phases: a ferromagnetic phase with $\left\langle\sigma^{z}\right\rangle>0$ and paramagnetic phase with $\left\langle\sigma^{z}\right\rangle=0$. From what we know of the $\Gamma=0$ and $\Gamma \rightarrow+\infty$ limits we can identify the first one with a dimer solid and the second one with the liquid. From the Ising point of view the solid phase is characterized by $\left\langle\sigma^{z}\right\rangle>0$. The two ferromagnetic Ising states $\left(\left\langle\sigma^{z}\right\rangle= \pm 1\right)$ correspond to the same dimer state on a closed surface but differ along the edge for open systems. The Ising magnetization $\left\langle\sigma^{z}(h)\right\rangle$ provides a non-local order parameter for the dimer solid, $\langle\Omega(h, \infty)\rangle$, which involves a string going to infinity.

Up to a sign, $\sigma^{z}(h)$ is the sum of a creation and annihilation operators of a vison. The dimer solidification can therefore be interpreted as the onset of off-diagonal order and macroscopic occupation number (of the zero-momentum state) for the visons. This QDM realizes a condensation of topological defects ("kinks" 18, 19] or visons [5]) at the confinement transition.
Notice that although we call it a solid, the large- $J$ phase does not go with a spatial spontaneous symmetry breaking since the $J$ part of $\mathcal{H}_{1}$ depends on an arbitrary reference state through the $\sigma^{z}$ operators; this term acts as an external potential which tends to pin the dimers along the reference state.

$\mathbb{Z}_{2}$ gauge theory. - The model of Eq. 3 is the dual of a $\mathbb{Z}_{2}$ gauge theory [20] (in its Hamiltonian formulation) where the gauge degrees of freedom $\tau^{z}(i)$ live on the bonds of $H$ (i.e. sites of $K)$. By definition $\tau^{z}(i)$ is the operator which flips the arrow at site $i$ and gauge-invariant observable are made of products of $\tau^{z}$ around closed loops. Spatial gauge transformations require the $x$ component, which we define with respect to the reference state $\left|D_{0}\right\rangle: \tau^{x}(i)=1$ if the arrow $i$ has the same orientation as in $\left|D_{0}\right\rangle$ and $\tau^{x}(i)=-1$ otherwise. For every site of $H$ (every triangle of $K$ ) the constraint reads $\tau^{x}\left(i_{1}\right) \tau^{x}\left(i_{2}\right) \tau^{x}\left(i_{3}\right)=1$ where $i_{1}, i_{2}$ and $i_{3}$ are the bonds of $H$ emanating from that site. This expresses the fact that physical states must be gauge invariant. This shows a one-to-one correspondence between physical state of the gauge theory and dimer coverings of $K$ and the redundancy in the gauge theory is solved by the dimer coverings. We wish to express $\mathcal{H}_{0}$ with the gauge degrees of freedom. Since $\sigma^{x}(h)$ operator flips all the arrows around $h$, it becomes the plaquette operator:

$$
\sigma^{x}(h)=\prod_{i} \tau^{z}(i)
$$

where the product runs over the bonds of $H$ surrounding $h$. The Ising interactions can be written

$$
\sigma^{z}(h) \sigma^{z}\left(h^{\prime}\right)=\tau^{x}(i)
$$

where $i$ is the common link between $h$ and $h^{\prime}$ (see Fig. 1). As a result the QDM of Eq. 3 translates into the Hamiltonian of a $\mathbb{Z}_{2}$ gauge theory (in continuous time), which is a manifestation of the well-known duality between $\mathbb{Z}_{2}$ gauge theories and Ising models $\left[19,20\right.$. Such a $\mathbb{Z}_{2}$ gauge theory has a confined and deconfined phases. A classical result is that they can be distinguished through the expectation value of a gauge-invariant Wilson loop [19, 20]

$$
W(\omega)=<\prod_{i \in \partial \omega} \tau^{z}(i)>=<\prod_{i \in \omega} \sigma^{x}(i)>
$$

where the first product runs over a close loop $\partial \omega$ which surrounds the area $\omega$. $W(\omega)$ changes from a perimeter law $\sim \exp (-|\partial \omega|)$ to an area law $\sim \exp (-|\omega|)$ when going from the deconfined at large $\Gamma$ to the confined phase at large $J$. The right-hand side of Eq. 6 expresses $W(\omega)$ as a correlation function for the dimer problem. $W(\omega)$ moves dimers along the loop $\partial \omega$ and the mapping to the gauge theory tells us that its long-distance behavior characterizes the liquid and frozen phases.

Liquid - crystal transition.- $\mathcal{H}_{1}$ has the disadvantage of depending on a specific reference state. To remedy for that 
we introduce a QDM which is reference free and restores the full lattice symmetry:

$$
\mathcal{H}_{2}=\mathcal{H}_{0}-J \sum_{h} s^{z}(h)
$$

where $s^{z}(h)= \pm 1$ is diagonal in the dimer basis and counts a factor -1 per anticlockwise arrow around the hexagon $h$. In the $J \rightarrow \infty$ limit the system selects $s^{z}(h)=1$ everywhere. This can be achieved if the system spontaneously breaks the translation symmetry and crystallizes in an ordered pattern of six-dimer "stars" described in Ref. [21]. These ordered states are degenerate with others $\left(\sim 2^{3 L}\right.$ where $L$ is the linear size) obtained by shifting the dimers along any straight line.

We have looked numerically (diagonalizations up to 54 kagome sites) at $\mathcal{H}_{2}$ and found evidence for a single and continuous transition from the liquid to the star crystal at $J \simeq \Gamma$. This appears as a collapse of the first excitation of the liquid, which then transforms into a degenerate groundstate of the crystal phase. We claim that the critical point is exactly at $J=\Gamma$ from a duality argument. From the arrow representation it is clear that $\sigma^{x}(h)$ and $s^{z}\left(h^{\prime}\right)$ commute except if $h$ and $h^{\prime}$ are neighbors, in which case they anticommute. Indeed the algebraic relations of $\sigma^{x}$ and $s^{z}$ are completely symmetric. In particular, we observed numerically that the ground-state energy is exactly symmetric with respect to the exchange of $\Gamma$ and $J$ (this is not true for all the eigenstates) and the critical point must lie at the self-dual point $J=\Gamma$.

Conclusions. - The kagome lattice has the remarkable property that dimer coverings correspond to the physical states of an Ising model on the triangular lattice and, by duality, of a $\mathbb{Z}_{2}$ gauge theory on the hexagonal one. Exploiting this, we introduced QDMs for which exact results are derived. In particular, we obtained for the first time the full spectrum of a QDM realizing a dimer liquid phase. It explicitly realizes fractionalized excitations and topological order. Through several models we showed that QDM on the kagome lattice are very simple and natural tools to investigate the connexions between frustrated magnets, RVB physics and spin-charge separation.

Acknowledgments. - We are grateful to M. Gaudin, K. Mallick, R. Moessner, C. Lhuillier and M. Feigel'man for several fruitful discussions. Numerical diagonalizations of QDM models were done on the Compacq alpha server of the CEA under project 550 .

\footnotetext{
* Electronic address: gmisguich@cea.fr
}

[1] D. S. Rokhsar and S. A. Kivelson. Phys. Rev. Lett. 61, 2376 (1988).

[2] P. W. Anderson, Science 235, 1196 (1987).

[3] R. Moessner and S. L. Sondhi, Phys. Rev. Lett. 86, 1881 (2001).

[4] X. G. Wen, Phys. Rev. B 44, 2664 (1991).

[5] T. Senthil and M. P. A. Fisher. Phys. Rev. Lett. 86, 292 (2001). Phys. Rev. B 63, 134521 (2001).

[6] A. Kitaev, quant-ph/9707021 (unpublished).

[7] L. B. Ioffe, M. V. Feigel'man, A. Ioselevich, D. Ivanov, M. Troyer and G. Blatter. Nature 415, 503 (2002).

[8] C. Nayak and K. Shtengel, Phys. Rev. B 64, 064422 (2001).

[9] L. Balents, M. P. A. Fisher, S. M. Girvin, Phys. Rev. B 65, 224412 (2002). Although dealing with a spin model on the kagome lattice, the solvable model discussed by these authors is closely related to that of Ref. [3] with dimers on the triangular lattice.

[10] B. Sutherland, Phys. Rev. B 37, 3786 (1988).

[11] N. Read and B. Chakraborty, Phys. Rev. B 40, 7133 (1989). S. Kivelson, Phys. Rev. B 39, 259 (1989).

[12] R. Moessner, S. L. Sondhi, and E. Fradkin. Phys. Rev. B 65, 024504 (2002).

[13] A. J. Phares and F. J. Wunderlich, Nuovo Cimento B 101, 653 (1988). V. Elser. Phys. Rev. Lett. 62, 2405 (1989).

[14] V. Elser and C. Zeng. Phys. Rev. B 48, 13647 (1993).

[15] C. Zeng and V. Elser. Phys. Rev. B 51, 8318 (1995).

[16] R. Siddharthan and A. Georges. Phys. Rev. B 65, 014417 (2002).

[17] Ch. Waldtmann, H. Kreutzmann, U. Schollwöck, K. Maisinger, and H.-U. Everts, Phys. Rev. B 629472 (2000).

[18] E. Fradkin and L. Susskind, Phys. Rev. D 17, 2637 (1978).

[19] J. B. Kogut, Rev. Mod. Phys. 51, 659 (1979).

[20] F. J. Wegner, J. Math. Phys. 12, 2259 (1971).

[21] A. V. Syromyatnikov and S. V. Maleyev, cond-mat/0202117 (unpublished).

[22] The QDM discussed in this work do not aim at describing the physics of the spin- $\frac{1}{2}$ kagome antiferromagnet. We are currently investigating a different QDM for this later problem.

[23] Dimer coverings are grouped in topological sectors by considering their transition graphs, that is the set of loops obtained by superposing two coverings on top of each other. Two coverings with only topologically trivial loops are in the same sector.

[24] EZ pseudospins variables are different from the well-known mapping [M. E. Fisher, J. Math. Phys. 7, 1776 (1966)] used to solve the Ising model on a planar lattices in terms of dimers.

[25] Up to a global sign (reference dependent) $\Omega(a, b)$ is equal to $\sigma^{z}(a) \sigma^{z}(b)$. 